\title{
Análise dos Coeficientes Piezoresistivos Fundamentais do Silício Policristalino
}

\section{Luiz Antonio Rasia ${ }^{1}$}

Universidade Regional do Noroeste do Estado do Rio Grande do Sul, UNIJUI, Ijuí, RS, Departamento de Ciências Exatas e Engenharias, DCEEng, Ijuí, RS

\section{Marina Geremias ${ }^{2}$}

Departamento de Ciências Exatas e Engenharias, DCEEng, Ijuí, RS

\section{Antonio Carlos Valdiero}

Universidade Regional do Noroeste do Estado do Rio Grande do Sul, UNIJUI, Ijuí, RS, Departamento de Ciências Exatas e Engenharias, DCEEng, Ijuí, RS

\begin{abstract}
Resumo. Neste trabalho são mostrados os coeficientes piezoresistivos longitudinal, transversal e de cisalhamento para filmes finos de silício policristalino tipo $\mathrm{N}$ e tipo $\mathrm{P}$ obtidos por simulação matemática. Estes coeficientes foram avaliados considerando o plano (100) e usando soluções analíticas estabelecidas para diafragmas circulares. Com base nos coeficientes piezoresistivos fundamentais, o efeito piezoresistivo em uma orientação cristalográfica arbitrária pode ser estimado. Este efeito desempenha um papel importante para o cálculo da sensibilidade dos elementos sensores quando se projeta piezoresistores de filmes finos que usam o silício como substrato.
\end{abstract}

Palavras-chave. Modelagem Matemática, Elementos Sensores, Piezoresistores, Coeficientes Piezoresistivos

\section{Introdução}

O efeito da piezoresistividade consiste na alteração da resistência elétrica de um material quando sobre ele é aplicada uma determinada tensão mecânica. Os dispositivos fabricados a partir deste conceito modulam a corrente elétrica em resposta a um sinal mecânico e são chamados de dispositivos piezoresistivos, necessitando de uma fonte externa de energia para funcionar em oposição aos dispositivos piezoelétricos.

A resistividade de um sólido não estressado é isotrópica, caso de estruturas cúbicas, já a variação de resistividade com a deformação mecânica é anisotrópica e descrita pela Eq. (1).

$$
\frac{\Delta \rho_{i j}}{\rho}=\sum_{k l} \pi_{i j k l} \sigma_{k l}
$$

\footnotetext{
1 rasia@unijui.edu.br

2 marinageremia@yahoo.com.br

3 valdiero@unijui.edu.br
} 
O tensor de piezoresistência tem a forma da Eq. (2).

$$
\frac{\Delta \rho_{i j}}{\rho}=\left(\begin{array}{cccccc}
\pi_{11} & \pi_{12} & \pi_{12} & 0 & 0 & 0 \\
\pi_{12} & \pi_{11} & \pi_{12} & 0 & 0 & 0 \\
\pi_{11} & \pi_{12} & \pi_{11} & 0 & 0 & 0 \\
0 & 0 & 0 & \pi_{44} & 0 & 0 \\
0 & 0 & 0 & 0 & \pi_{44} & 0 \\
0 & 0 & 0 & 0 & 0 & \pi_{44}
\end{array}\right)\left(\begin{array}{l}
\sigma_{11} \\
\sigma_{22} \\
\sigma_{33} \\
\sigma_{12} \\
\sigma_{23} \\
\sigma_{31}
\end{array}\right)
$$

Em materiais semicondutores existem diferentes tipos de estruturas de banda com diferentes formas de superfícies de energia no espaço de onda, K. Estas superfícies se modificam e promovem uma mudança da resistividade, $\rho$, do material. Este processo físico é denominado de intervales [8] e ilustrado na Figura 1.
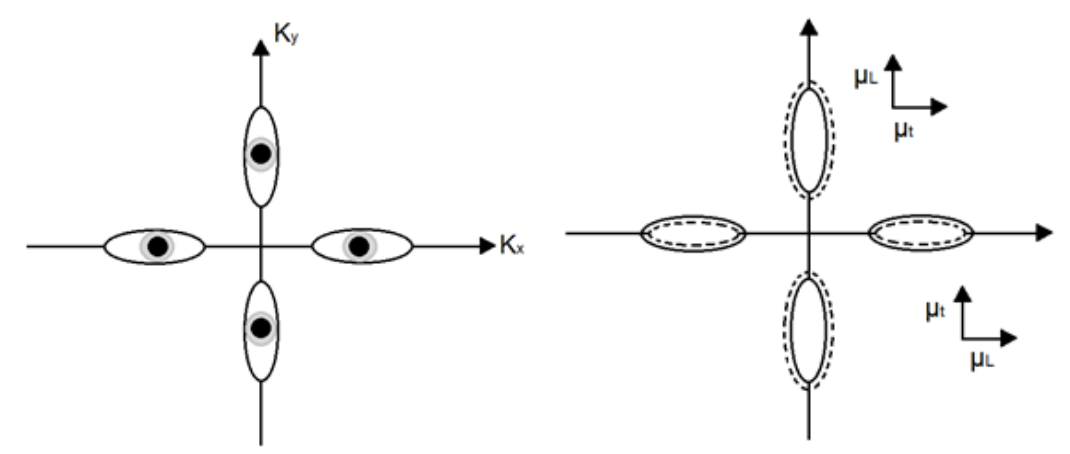

Figura 1: Ilustração das superfícies de energia para o silício não estressado e estressado.

Estes conceitos são fundamentais para a fabricação de dispositivos sensores, como strain gauges, acelerômetros, sensores de pressão.

\section{Materiais Semicondutores e Modelo de Piezoresistor}

Nestes últimos anos muitos tipos de materiais têm sido investigados para uso em dispositivos sensores, em especial, em dispositivos de pressão. Entretanto, o silício ainda é o material mais empregado nestas aplicações devido ao domínio dos processos de fabricação e a combinação de suas excelentes propriedades.

Basicamente, divide-se o silício em monocristalinos e policristalinos, os quais possuem propriedades piezoresistivas importantes, cada um com suas particularidades. Assim, o silício monocristalino está limitado a trabalhar em ambientes de temperatura não muito elevada devido aos efeitos de junção embora apresente um gauge factor ou fator de sensibilidade muito elevado quando comparado com outros materiais.

De outro modo, o silício policristalino não apresenta efeitos de junção porque os piezoresistores ficam isolados por uma camada de óxido. Desse modo, podem trabalhar em altas temperaturas e ter um bom fator de sensibilidade aos esforços mecânicos se comparado com os piezoresistores fabricados com silício monocristalino.

A concentração de impurezas, $\mathrm{N}$, é usada para alterar a condutividade dos semicondutores de modo que os mesmos podem se tornar materiais tipo $\mathrm{N}$ ou tipo $\mathrm{P}$, 
dependendo do dopante como sugere a literatura [4].

Os coeficientes piezoresistivos, $\pi \mathrm{s}$, para material tipo $\mathrm{P}$ e tipo $\mathrm{N}$, estão relacionados com os níveis de concentração de impurezas, $\mathrm{N}$, orientação cristalográfica do material, temperatura e, consequentemente, com o tipo de condutividade [1], conforme ilustram as Figura 2a) e Figura 2b).
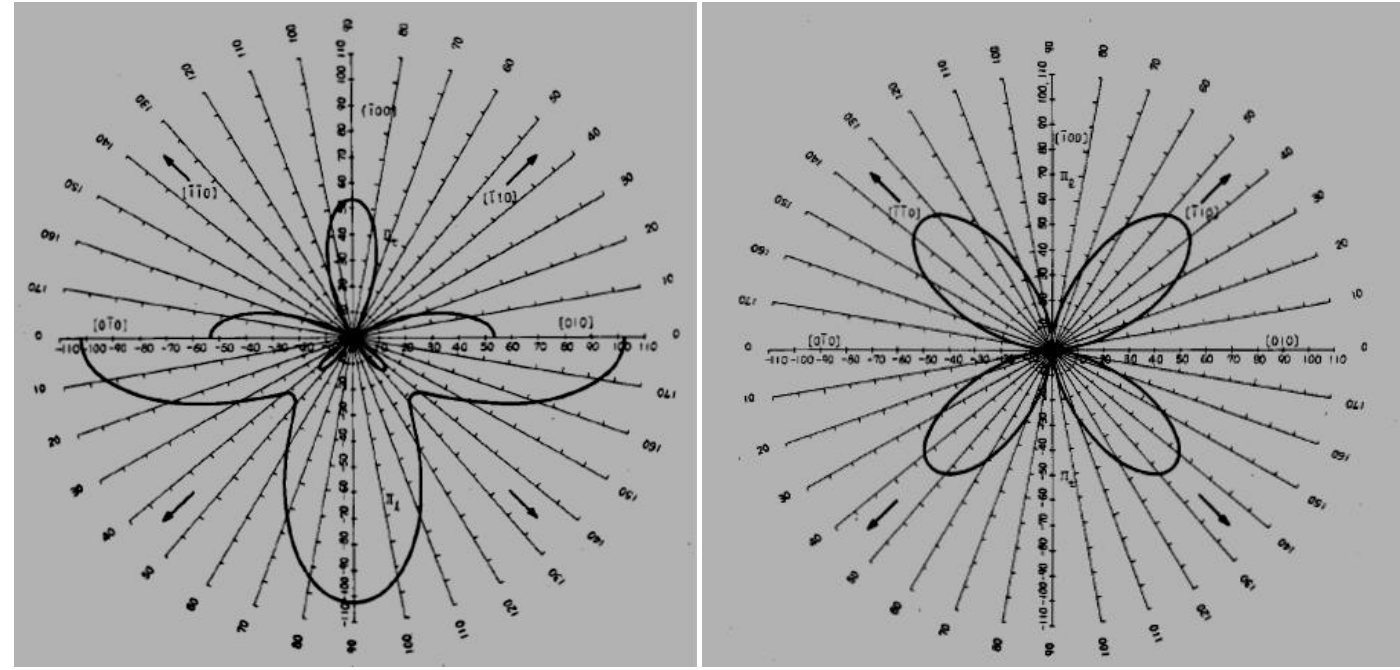

Figura 2: a) Coeficiente piezoresistivo longitudinal para o material tipo $\mathrm{N}$ [4]. b) Coeficiente piezoresistivo longitudinal para o material tipo $\mathrm{P}[4]$.

Estes parâmetros são importantes para os processos tecnológicos de fabricação dos dispositivos sensores e atuadores uma vez que estão relacionados com a sensibilidade dos dispositivos $[3,5,7]$.

Neste trabalho, serão estudados elementos sensores com as seguintes dimensões geométricas: $40 \mu \mathrm{m}$ de largura e comprimentos variando de $40 \mu \mathrm{m}$ à $100 \mu \mathrm{m}$ e resistividade da ordem de $0,0018 \Omega . \mathrm{cm}$, feitos de silício policristalino. O modelo físico destes elementos sensores é mostrado na Figura 4 [3].

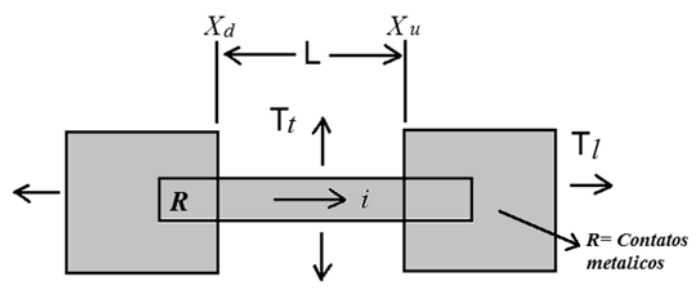

Figura 4: Modelo Físico de Piezoresistor.

O modelo matemático que descreve esta variação da resistência elétrica pode ser reescrito e relacionado com os componentes longitudinais e transversais do estresse mecânico e com os respectivos coeficientes piezoresistivos do material de acordo com a 
Equação (3).

$$
\frac{\Delta \mathrm{R}}{\mathrm{R}}=\pi_{\mathrm{l}} \mathrm{T}_{\mathrm{l}}+\pi_{\mathrm{t}} \mathrm{T}_{\mathrm{t}}
$$

Sendo que $\pi_{1}$ e $\pi_{\mathrm{t}}$ são os coeficientes piezoresistivos longitudinais e transversais, e, $\mathrm{T}_{1}$ e $T_{t}$ são os esforços mecânicos longitudinais e transversais ao eixo de aplicação da força sobre o cristal semicondutor.

Um modelo de piezoresistor que considera efeitos de temperatura pode ser descrito pela Equação (4).

$$
R(P, \theta)=R_{r e f}(\theta)\left[1+\pi_{l l}(\theta) T_{l l}(P, \theta)+\pi_{t}(\theta) T_{t}(P, \theta)+\pi_{x y}(\theta) T_{x y}(P, \theta)\right]
$$

Onde, $\mathrm{P}$ é a pressão aplicada, $\theta$ é a temperatura, $\mathrm{R}_{\text {ref }}$ é a resistência sem esforço mecânico na temperatura de referência $(300 \mathrm{~K}), \pi_{\mathrm{xy}}$ é o coeficiente piezoresistivo de cisalhamento no plano x-y e $\mathrm{T}_{\mathrm{xy}}$ é o esforço mecânico de cisalhamento médio [3].

O diagrama ilustrado na Figura 5 mostra como um material com propriedades semicondutoras se comporta em termos elétricos, mecânicos e térmicos [7]. A partir desta análise inicia-se o projeto de um transdutor.

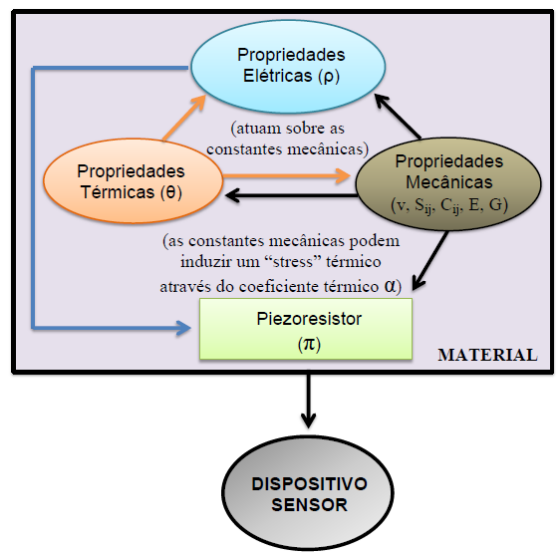

Figura 5: Efeitos cruzados em Piezoresistores.

O sinal e a magnitude do efeito piezoresistivo está associada com o tipo de dopante, temperatura, orientação e qualidade cristalográfica do material escolhido [5]. Portanto; o posicionamento dos piezoresistores sobre diafragmas devem ser alinhados com grande precisão, em regiões de máximo esforço mecânico, visando aperfeiçoar os efeitos resultantes do cruzamento das propriedades do material [7].

\section{Modelamento dos Coeficientes Piezoresistivos Fundamentais de Elementos Sensores}

O conhecimento do coeficiente piezoresistivo longitudinal, $\pi_{\mathrm{l}}$, transversal, $\pi_{\mathrm{t}}$, e de 
cisalhamento $\pi_{\mathrm{s}}$ de um material, é importante para a fabricação de um piezoresistor otimizado. Os coeficientes são determinados a partir do emprego de relações trigonométricas, de acordo com a Equação (5), Equação (6) e Equação (7).

$$
\begin{aligned}
& \pi_{l}=\pi_{11}-\frac{1}{2}\left(\pi_{11}-\pi_{12}-\pi_{44}\right) \sin ^{2} 2 \theta \\
& \pi_{t}=\pi_{12}+\frac{1}{2}\left(\pi_{11}-\pi_{12}-\pi_{44}\right) \sin ^{2} 2 \theta \\
& \pi_{s}=-\frac{1}{2}\left(\pi_{11}-\pi_{12}-\pi_{44}\right) \sin 4 \theta
\end{aligned}
$$

Os coeficientes piezoresistivos $\pi_{11}$ e $\pi_{12}$ são correspondentes às tensões mecânicas, $\mathrm{T}_{\mathrm{x}}, \mathrm{T}_{\mathrm{y}}$ aplicadas na direção uniaxial [100], na qual a banda de deformação é insignificante e o mecanismo dominante, do efeito piezoresistivo, é a mudança na taxa de espalhamento das cargas elétricas. Por outro lado, o coeficiente piezoresistivo $\pi_{44}$ está associado à tensão uniaxial, $\mathrm{T}_{\mathrm{xy}}$, na orientação [110], na qual a superfície de energia é significativamente deformada, o que tem como consequência à mudança da massa efetiva dos portadores de carga. Assim, na orientação [110] a tensão mecânica produz efeito piezoresistivo mais expressivo [4].

\section{$4 \quad$ Resultados e Simulações}

O comportamento dos coeficientes piezoresistivos longitudinais, $\pi_{\mathrm{l}}$, transversais, $\pi_{\mathrm{t}}$, e de cisalhamento, $\pi_{\mathrm{s}}$, em uma orientação cristalográfica arbitrária do plano (100) para polissilício tipo P é mostrado na Figura 6.

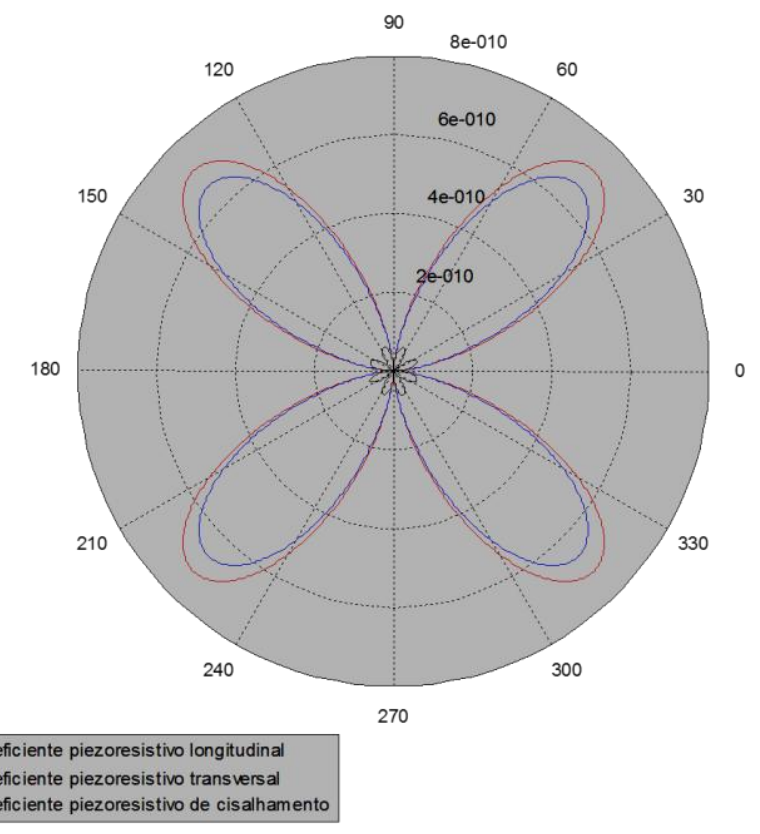

Figura 6: Comportamento dos coeficientes piezoresistivos longitudinal, transversal e de 
cisalhamento do filme de polissilício tipo P no plano (100).

Os valores dos coeficientes piezoresistivos máximos para os piezoresistores de polisilício tipo P são, $\pi_{l}=7,18 \times 10^{-10} \mathrm{~m}^{2} / N, \pi_{t}=-6,63 \times 10^{-10} \mathrm{~m}^{2} / N$ e $\pi_{44}=6,52 \times 10^{-11} \mathrm{~m}^{2} / \mathrm{N}$, respectivamente.

Verifica-se, na Figura 6, que os valores dos coeficientes longitudinal e transversal apresentam a mesma ordem de magnitude enquanto que os valores do coeficiente de cisalhamento são da ordem de dez vezes menor.

A Figura 7 mostra os valores dos coeficientes piezoresistivos máximos para os piezoresistores de polisilício tipo $\mathrm{N}$ sendo, $\pi_{l}=-3,12 \times 10^{-10} \mathrm{~m}^{2} / \mathrm{N}$, $\pi_{t}=-1,76 \times 10^{-10} \mathrm{~m}^{2} / N$ e $\pi_{44}=7,1 \times 10^{-11} \mathrm{~m}^{2} / N$, respectivamente.

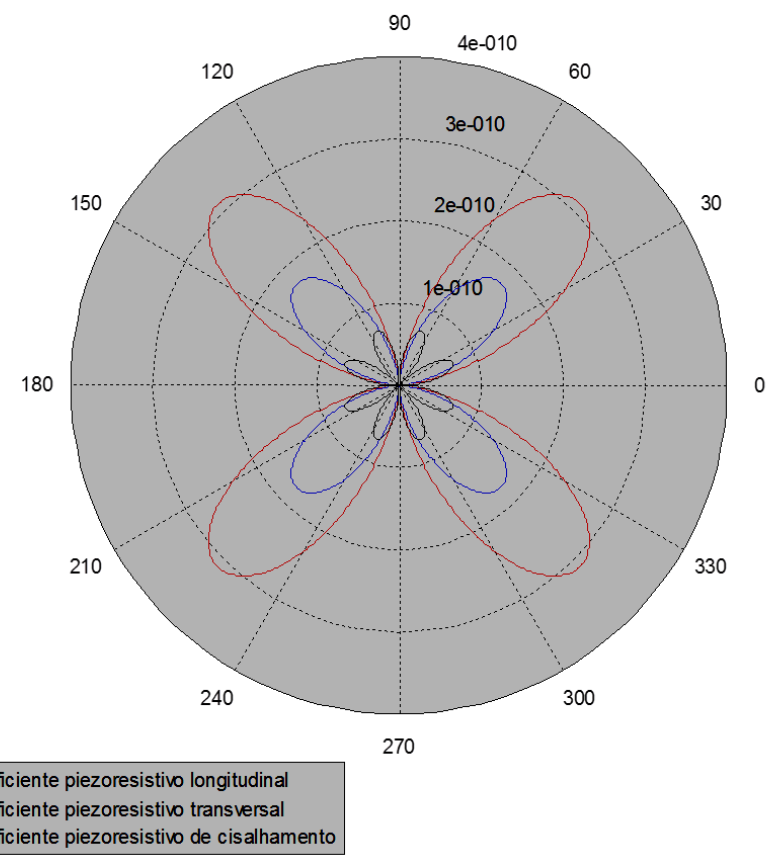

Figura 7: Comportamento dos coeficientes piezoresistivos longitudinal, transversal e de cisalhamento do filme de polissilício tipo $\mathrm{N}$ no plano (100).

Os resultados mostrados na Figura 6 e Figura 7 apresentam um perfil muito semelhante aos apresentados por [6], para filmes de 3C-SiC. Portanto; o método de análise empregado, neste trabalho, permite obter os coeficientes piezoresistivos de forma satisfatória.

\section{Conclusões}

Neste trabalho são evidenciadas diferenças significativas nos resultados obtidos para os coeficientes piezoresistivos dos filmes de polisilício analisados. A análise gráfica mostra a influência dos mecanismos de condução elétrica nos filmes semicondutores e servem como indicativos para projetos de elementos sensores piezoresistivos otimizados elétrica, térmica e mecanicamente. 


\section{Agradecimentos}

Os autores agradecem a Unijui pelo uso dos laboratórios e a CAPES pela bolsa de pesquisa.

\section{Referências}

[1] M. Akbar, Interface circuit for piezoresistive pressure sensors, Dissertation, Department of Electrical Engineering, University of Michigan, (1991).

[2] H. Furlan, Proposta de implementação de membranas em sensors de pressão a elementos piezoresistivos utilizando a técnica de pós-processamento, Dissertação de mestrado, EPUSP, (1997).

[3] Z. Gniazdowiski, P. Kowalski, Pratical approach to extraction of piezoresistance coeffient, Institute of Electron Technology, Warsaw, Poland (1998).

[4] Y. Kanda, A graphical representation of the piezoresistance coefficients in silicon, IEEE Transactions on electron devices, vol. Ed-29, (1982).

[5] L. Maisel, R, Glang, Handbook of thin film technology, McGraw Hill Company, (1983).

[6] H. P. Phan, D. V. Dao, P. Tanner, L. Wang, N. T. Nguyen, Y. Zhu, S. Dmitrijev, Fundamental piezoresistive coefficients of p-type single crystalline 3C-SiC, Appl. Phy. Let., 104, (2014).

[7] L. A. Rasia, Elementos Piezoresistivos para sensors de pressão com tecnologia CMOS, Dissertação de mestrado, EPUSP, (1997).

[8] L. H. Vlack, Propriedades de materiais cerâmicos, Edgard Blücher, (1973). 